\title{
Effects of Cortical and Subcortical Stimulation on Delayed Response in Monkeys
}

\author{
S. N. Cianci ${ }^{1}$ \\ Department of Psychology, The University of Michigan, Ann Arbor, Michigan
}

Received July 16, 1964

\begin{abstract}
Sixty-four brain points in five monkeys were tested for the effect of electrical stimulation during the associative period and the retentive period of a delayed response task. Stimulation within the limbic and lower extrapyramidal. systems resulted in marked impairment of delayed response performance. Deficits were usually obtained during both the predelay and delay intervals. On the other hand stimulation of loci in cortex and basal ganglia resulted primarily in differential effects on association formation and retention. No impairment was seen from many probes within the latter areas.
\end{abstract}

\section{Introduction}

A long standing question in the physiology of higher mental functions has been whether the processes of learning and memory are served by the same or different neural systems. Attempts to separate the processes by ablation have provided a great deal of information, but have not been successful in regard to the basic question.

Bilateral resection of the prefrontal lobes of monkeys results in a loss of the animals' ability to perform the delayed response test (6). The task, which is the classical tool for measuring brief retention following an associative input in monkeys, has three basic intervals; an associative, a retentive and a performance interval. During the associative phase critical cueing takes place; during the retentive phase the problem table is hidden from view, and during the performance phase the animal

1 This work is based on a dissertation submitted in partial fulfillment of the requirements for the Ph.D. degree at the University of Michigan. The research was supported by grants from the U. S. Public Health Service and National Science Foundation awarded to Prof. J. Olds. The author gives his thanks to Professor Olds for his valuable consultations. Thanks are also due to Dr. R. E. Myers and Dr. J. Finan for their constructive criticisms of the manuscript. Current address: Laboratory of Neurological Sciences, Spring Grove State Hospital, Baltimore, Maryland. 
is allowed to respond but in the absence of the critical cue. The loss seen with prefrontal lesions has been attributed to diverse causes implicating both the associative and retentive processes $(7,12)$. Moreover, by altering the experimental conditions (9) or the state of the animal (16) the effects of the lesion appear to be compensated for, suggesting that other brain areas may be involved as well.

Deficits in dclayed alternation performance, a task in many regards similar to delayed response, have been reported with lesions in the caudate nucleus (13). Electrical stimulation, a technique which permits the experimenter to produce reversible lesions, was demonstrated by these investigators to cause deficits comparable to those seen with electrocoagulation of the caudate nucleus. Stamm (14), also using electrical stimulation, found that excitation of prefrontal areas impaired mastery of delayed alternation but did not effect performance in animals which had been overtrained. The same result had been noted with epileptogenic foci (15).

Weiskrantz, Mihailovic and Gross (18) demonstrated that stimulation was an effective disrupter of delayed alternation ability when applied along the banks of the principal sulcus; stimulation along nearby arcuate sulcus in the prefrontal area proved ineffective. These workers also demonstrated that unilateral stimulation, though not as efficacious as bilateral excitation, was an effective disrupter. Since unilateral resections have no effect, electrical stimulation may be a more sensitive indicator of critical foci.

In the present study diverse brain areas were sampled by means of electrical stimulation to determine critical foci. The stimulation was applied differentially during the associative and retentive phases of a delayed response task in an effort to determine whether there may be systematic differences within or between the sampled regions in relation to the stimulation interval.

\section{Method}

Subjects and Apparatus. Five macaque monkeys served as subjects. Three were young adult males ( $M$. cynomologous), $A l, F r$, and $T i$; one was an immature male (M. mulatta), $R h$; and one was a young adult female ( $M$. nemestrina), $J u$.

The animals were permanently seated in primate chairs modified to include components of the Wisconsin General Test Apparatus. The problem surface had two centrally located food-wells $8 \mathrm{~cm}$ apart. The wells 
were covered by identical lids which could be separately pushed by the animal to expose the contents. Two barriers were suspended by pulleys between the animal and the wells; a transparent one to permit the subject to view the baiting and an opaque one to hide the wells from view during the retention period. The two shields could be raised and lowered independently or together. The animals could respond only when both were raised.

Preoperative Training. The animals were trained in a left-right delayed response task, direct method, with a 5-sec delay period. A Gellerman series determined the sequence of side baitings. Correction technique and shorter delays were used in the early phases of the training. Noncorrection technique was introduced as the animal showed signs of mastering the problem. Incorrect responses were not punished except by the absence of the fruit reward and an occasional accidental rap on the knuckles by the descending doors. The criterion was met by the subject scoring at least $90 \%$ correct on four consecutive blocks of fifty trials each with the noncorrection method. All animals except $R h$ were trained preoperatively.

Preliminary Postoperative Testing. After a 2-week recovery period, the brain points were stimulated to determine movement thresholds and to note other behavioral changes. A 60-cycle/sec sine wave pulse of 1-2 sec duration was delivered monopolarly in these determinations. Stimulation was monitored on a cathode ray oscilloscope. Animals were then run on the delayed response task with the electrode leads in place and the stimulator on, but set at zero intensity, to allow the animals to habituate to the experimental conditions. The animals adapted quickly and the experiment proper was begun.

The Experiment. The animals were tested daily under three conditions. Two of the conditions involved stimulation while the third served as a control. Stimulation parameters used for each point were below the threshold value previously determined.

Each served as its own control. The animals were maintained on a 12-hour food deprivation schedule during the testing. One brain point was studied under all conditions on a given day. The animal was run a minimum of fifty trials per condition and there was a 45 -min interval between conditions. Brain points were restudied in the same fashion, though sometimes at different amperages, at later dates. The sequence of conditions was varied from day to day to eliminate order effects.

Condition I. Stimulation during the associative period. A 60 -cycle/sec sine wave stimulus consisting of 0.5 -sec trains at 1 per sec was applied 
for $5 \mathrm{sec}$ during the baiting period. Stimulation for cach trial began when the animal's attention was directed to the bait being held over the uncovered food-well. During the stimulation the food was dropped into the well and the well covered. After $5 \mathrm{sec}$ the stimulation ceased and the opaque barrier was lowered. Thus the last view the animal had through the transparent shield was of two identical food-well lids. Stimulation during this period might act to prevent the animal from forming the association between the bait and the right or left food-well.

Condition II. Stimulation during the retentive period. The animal was allowed to view the baiting procedure without stimulation, but, with the lowering of the opaque barrier, $5 \mathrm{sec}$ of stimulation commenced. The stimulation was identical in all respects to that applied during Condition I. At the end of the 5 -sec delay period the pulsations ceased and both barriers were raised. Stimulation always ended prior to the animal's response, thereby eliminating any possibility of the motor response, as such, being directly disturbed. Stimulation during the retention period might act to cause the animal to lose the association formed during the baiting period.

Condition III. No stimulation. Control trials were run daily with the electrode leads in place but with the stimulator set at zero intensity. In addition, immediately upon completion of each stimulation bloc and without interruption of the routine, ten additional control trials were run. These poststimulatory control trials were used as an index of the aftereffects of stimulation.

Histology. Upon completion of all procedures the animals were anesthetized and perfused with saline followed by $10 \%$ formalin. Electrode locations were determined from frozen sections sliced at $50 \mu(2,11)$.

\section{Results}

Sixty-four brain points located in thirty-three structures in the five subjects were studied under the three conditions. An analysis of the behavioral data from the individual points demonstrated six major groupings.

a. Points where stimulation caused no effect. Brain foci, where stimulation did not significantly alter the animal's score on the delayed response test, were located predominantly in cortex and basal ganglia, although some probes fell within the thalamus and brain stem.

b. Points of adaptation. The effect produced by stimulation of these points changed with repeated trials. Typically a disruptive effect was seen during the early stimulation trials; it was usually mild though not always, 
and disappeared or diminished with repeated stimulation. Points demonstrating this effect were located predominantly within the thalamus and brain stem. Increasing the amperage sometimes returned the animal to the disrupted state but with continued stimulation the effect again abated. At some sites additional trials or an increase in amperage seemed to enhance the animal's performance (Fig. 1). The apparent enhancement is difficult to evaluate since the animals were quite adept and normally yielded a high percentage of correct responses. The adaptation was often

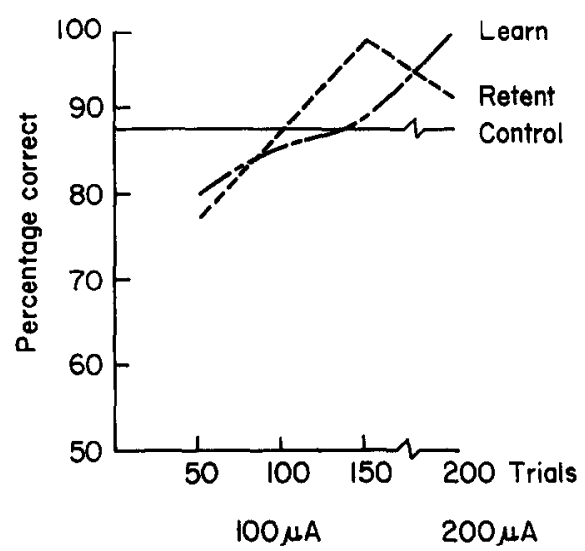

FIg. 1. Adaptation and apparent enhancement from stimulation of anterior medial thalamus.

restricted to stimulation during only one phase of the delay task. Increasing the amperage during additional trials sometimes resulted in a reduction of errors in one condition and an increase in errors in the other.

c. Points effected by stimulation during the associative interval. Some structures showed the greater effect due to stimulation during baiting. Occasionally there were milder, though sometimes significantly deleterious, effects seen in the other stimulation condition. Increasing the amperage sometimes produced a fairly uniform deficit in both conditions; nevertheless, the over-all impression was one that the stimulation predominantly impaired the animals' ability to form the association between the bait and the appropriate food cup. Electrodes exhibiting this effect were generally located in cortex (prefrontal and cingulate) and basal ganglia.

d. Points effected by stimulation during the retentive interval. Stimulation was more efficacious when applied during the delay interval, but deficits were seen in the other condition as well. In general, the degrees 
of deficits were comparable to those seen in the previous group. Electrodes exhibiting the effect of causing the animal to lose the formed association were also predominantly located in cortex and basal ganglia.

e. Points effected by stimulation during both intervals. This category is comprised of those areas where an effect was discernible during both phases. The degrees of deficits were more profound than those noted where the stimulation acted in a more differential manner. Electrodes demonstrating this uniform, severe impairment were located predominantly in limbic and lower extrapyramidal structures.

f. Inhibition of behavior with stimulation. Continued stimulation at some points caused the animals to refuse to respond. No response was defined by the animal's refusal to reach for the food-wells within $10 \mathrm{sec}$ on three consecutive trials. The animals typically showed an increase in errors accompanied by an increase in latency of response which finally culminated in total cessation of the operant behavior. In some instances the effect took place immediately precluding thorough study of the point, e.g., substantia nigra in $A l$; while at other loci the inhibition was not seen until the animal had been stimulated many times. The duration of the effect was also variable. On some days the animal's usual behavior could be reinstituted by turning off the stimulation while at other times this procedure failed. Also noted at these inhibitory points was an increase in the emotionality of the animal accompanied by a general regression in behavior. The animal would try to pull or lift the lids rather than push them, and would face away from the experimenter. These types of behaviors

TABLE 1

ANATOMICAL-BeHAVIORAL INTERACTION ${ }^{a}$

\begin{tabular}{lccccc}
\hline & Learn & Retent & Both & No effect & Total \\
\hline $\begin{array}{l}\text { Cortex } \\
\text { and } \\
\text { basal ganglia }\end{array}$ & 7 & 4 & 0 & 15 & 26 \\
$\begin{array}{l}\text { Limbic and } \\
\text { lower } \\
\text { extrapyramidal }\end{array}$ & 1 & 2 & 12 & 0 & 15 \\
$\begin{array}{l}\text { Thalamic } \\
\text { and } \\
\text { brain stem }\end{array}$ & 4 & 2 & 2 & 6 & 14 \\
\hline
\end{tabular}

a Tables listing the points falling into each behavioral category will be provided upon request addressed to the author. Ventricular and adaptation points are excluded from table. Chi square with $6 d f$ is $35.30, p<0.01$. 
were observed in the earliest phases of the training prior to the implantation.

The behavioral groupings were next analyzed from a gross anatomical viewpoint. Three broad categories suggested themselves: (i) Cortex (prefrontal and cingulate) and basal ganglia; (ii) limbic and lower extrapyramidal; and (iii) thalamic and brain stem. A chi square test $(4,5)$ based on the anatomical frequencies within the behavioral cells was highly significant indicating that brain points were distributed in the behavioral groupings in a differential rather than a random manner (Table 1).

Self-stimulation lest. Animal $R h$ was lested for self-stimulation prior to delayed response training in order to determine the role motivational areas may have on associative and retentive processes. The results of the stimulation of motivational areas indicated that as the amperage approached the threshold for self-stimulation the errors increased slowly, but as the threshold was crossed the errors increased sharply and the inhibition phenomenon was seen (Fig. 2).

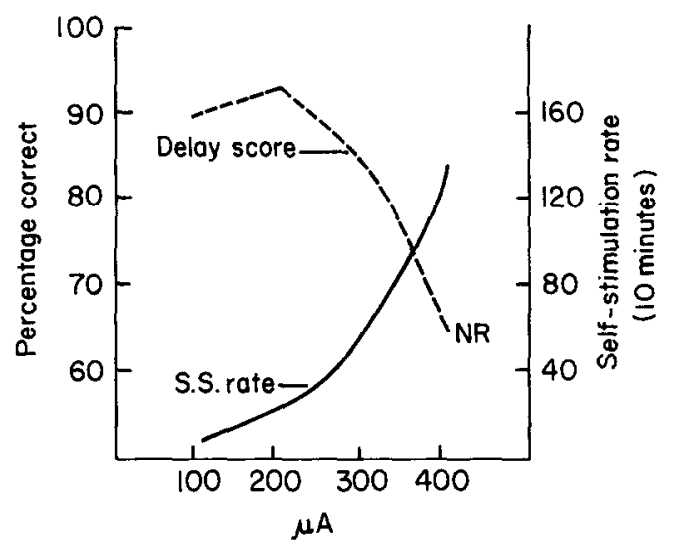

FIG. 2. Relationship between stimulation of positively reinforcing areas and delayed response score. "Retention" in septal nuclei.

\section{Discussion}

Stimulation of widespread brain foci resulted in a variety of deficits: At some sites deficits were seen predominantly with stimulation during association formation; at some, deficits were seen predominantly during retention; and at some, deficits were seen during both processes. While many structures were sampled none were studied in sufficient degree to warrant a detailed anatomical analysis of behavioral function; however, 
the pooling of foci according to general anatomical class revealed a relationship between the nature of the deficit seen and the type of tissue sampled.

Differential effects, i.e., deficits occuring during either the association formation or retention intervals, were primarily caused by the electrical excitation of prefrontal cortx or basal ganglia. Stimulation of many probes in this tissue was ineffective. The finding of effective and ineffective sites within the same gross region generally agrees with the results of Weiskrantz et al. (18) and is consistent with the focus-field effect seen in ablation studies. On the other hand, excitation of probes within limbic and lower extrapyramidal tissue always was an effective disrupter, in most instances during both the associative and retentive intervals, and only occasionally during one or the other. The occurrence of selective deficits caused by differentially applied stimulations suggests that the associative and retentive processes may be subserved by separate mechanisms within prefrontal cortical and basal ganglia complexes. These separate systems share not only the same general loci but produce comparable degrees of deficits when stimulated. Increasing the amperage caused general, rather than selective, deficits to appear indicating that a close inter-relationship exists between the systems. In this regard the tissue appears to be organized similarly to the sensory and motor areas where each function is represented within the scope of the other.

The functional systems hypothesized here are based on behavioral delayed response data. Walker, Poggio and Andy (17) have presented electrophysiological evidence for a functional organization of the brain into systems. They described three systems for the propagation of cortically induced epileptogenic discharges. These workers commented that afterdischarges were seen in the contralateral area, that there can be intersystem activation, and that discharges can spread to subcortical structures.

The electrical excitation, possibly, was being propagated in a similar fashion. The contralateral spread might explain the effectiveness of unilateral stimulation compared with the ineffectiveness of unilateral prefrontal ablations. In this study probes falling within the dorsal aspects of the caudate nucleus usually produced deficits when stimulated while those within the ventral aspects did not. Interestingly, the dorsal aspects of the caudate are related transcallosally while the ventral aspects are not (3).

Similarly, if stimulation of the dorsomedial thalamus was propagated throughout a system an effect would more likely be expected, as was found 
in this study, than would be the case if this structure alone were ablated (1). The uniformity of impairment noted with increased amperages in those areas where differential effects were previously noted suggests that inter-system propagation might be occurring. The poststimulatory depression of control scores noted at some loci also lends itself to a propagation interpretation. It is important to note that many brain points were not effected by stimulation indicating that insofar as propagation was occurring it was not occurring in a randomly diffuse manner.

The occurrence of nonselective deficits suggests that association and retention may be served by one system comprised essentially of limbic and lower extrapyramidal structures. Stimulation within this system always had an effect and of greater severity than that seen from the other anatomical class suggesting that the unitary system is a more primitive or powerful complex. The recovery of function seen in prefrontal animals with predelay reinforcement might be accounted for by this complex.

The limbic system has been implicated in emotional and associative behavior (10). In terms of complex learned behavior the role of the extrapyramidal system has not been well defined. However, it has been implicated in the "instinctive" behavior of animals having a negligible cortex but a well defined striatum (8).

While the anatomical groupings stipulated here certainly do injustice to morphologic relations they do have a functional relationship. Cortical and basal ganglia stimulation never effected both learning and retention; It either caused differential deficits or none. The limbic-lower extrapyramidal complex always had an effect, usually on both functional processes. The last anatomical class, thalamic and brain stem, is in many regards more diffuse than the other two. This class, as might be expected, was represented in all behavioral groups.

The anatomical data taken in conjunction with the behavioral data suggest that there is basically one system for both learning and retention. The core of this system might be comprised of structures common to both the extrapyramidal and limbic complexes. This system appears to be supplemented by two closely related subsystems, one for learning and one for retention. The subsystems might be comprised of cortical and striatal areas and might act to add refinement to the basic function.

The nature of the mechanism underlying the functions remains unanswered. It may be associative, retentive, motivational and interactive. A motivational component appeared to be related to the asso- 
ciative-retentive functions in this study. However, further work covering negative and ambivalent foci as well as positive ones is needed to explicate the motivational aspects.

The roles of nonreversible and reversible lesions must also be clarified. In this study the stimulation was seen to act in a number of ways. It appeared to be compeltely ineffective; to act as a neutral peripheral stimulus; to enhance the animal's ability; and to act as a lesion. When the stimulation was acting as a lesion the animals in this study were like their prefrontal counterparts in terms of delayed response score, but they were different in other respects. The prefrontal animal reputedly is not upset by errors and continues working despite failure. The stimulated animals quite often become agitated with errors and ceased responding. In ablated animals the errors have been attributed to a release from inhibition following the cortical loss. In stimulated animals errors and inhihition were often seen together. Interestingly, the inhibition was seen only with stimulation of subcortical structures pointing once again to the complexity of cortical and subcortical relations.

\section{References}

1. CHow, K. L. 1954. Lack of behavioral effects following destruction of some thalamic association nuclei. A.M.A. Arch. Neurol. Psychiat. 71: 762-771.

2. Clarke, R. H., and E. E. Henderson. 1920. Investigation of the central nervous system. Part II. Atlas of photographs of the frontal sections of the cranium and brain of the rhesus monkey (Macacus Rhesus), Special Volume Part 2, pp. 161-172 \& Plates. In "Johns Hopkins Hospital Reports" Lord Baltimore press, Baltimore, Maryland 21-57 (1920).

3. Ebner, F., and R. E. Myers. 1964. Distribution of the corpus callosum and anterior commissure in the monkey. J. Comp. Neurol. (in press).

4. Edwards, A. L. 1950. "Experimental Design in Psychological Research," Holt, New York.

5. Guilford, J. P. 1956. "Fundamental Statistics in Psychology and Education." McGraw-Hill, New York.

6. Jacobsen, C. F. 1935. Functions of the frontal association areas in primates. A.M.A. Arch. Neurol. Psychiat. 33: 558-569.

7. JacobSen, C. F. 1936. Studies of cerebral function in primates. Comp. Psychol. Monog. 13: 3-60.

8. Jung, R., and R. Hassler, 1960. The extrapyramidal motor system, pp. 863-927. In "Handbook of Physiology. Section I, Neurophysiology," Vol. II, Am. Physiol. Soc., Washington, D, C.

9. MaLmo, R. B. 1942. Interference factors in delayed response in monkeys after removal of frontal lobes. J. Neurophysiol. 5: 295-308.

10. Olds, M. E., and J. OLds. 1961. Emotional and associative mechanisms in rat brain. J. Comp. Physiol. Psychol. 54: 120-126. 
11. OLszewski, J. 1952. "The Thalamus of the Macaca Mulatta: An Atlas for Use with the Stereo-Taxic Instrument." Karger, Basel.

12. Pribram, K. H., and M. Mishrin. 1956. Analysis of the effects of frontal lesions in monkeys. IIT. Object alternation. I. Comp. Physiol. Psychol. 49: 41-45.

13. Rosvold, H. E., and J. M. R. Delgado. 1956. The effect on delayed alternation test performance of stimulating or destroying electrically structures within the frontal lobes of the monkey's brain. J. Comp. Physiol. Psychol. 49: $365-372$.

14. Stamm, J. S. 1961. Electrical stimulation of frontal cortex in monkeys during learning of an alternation task. $J$. Neurophysiol. 34: 414-426.

15. Stamm, J. S., and K. H. Pribram. 1960. Effects of epileptogenic lesions in frontal cortex on learning and retention in monkeys. J. Neurophysiol. 33: 552-563.

16. WADE, M. 1947. The effect of sedatives upon delayed responses in monkeys following removal of the prefrontal lobes. J. Neurophysiol. 10: 57-61.

17. Walker, A. E., G. F. Poccro, and O. H. ANDy. 1956. Structural spread of cortically induced epileptic discharge. Neurology 6: 616-626.

18. Weiskrantz, L., L. Murailovic, and D. G. Gross. 1962. Effects of stimulation of frontal cortex and hippocampus on behavior in the monkey. Brain 85: 487-504. 